Bone Health Assessment in Pediatrics 

Ellen B. Fung - Laura K. Bachrach

Aenor J. Sawyer

Editors

\section{Bone Health Assessment in Pediatrics}

Guidelines for Clinical Practice

Second Edition

望 Springer 


\section{Editors}

Ellen B. Fung

UCSF Benioff Children's Hospital Oakland

Children's Hospital Oakland Research Institute

Oakland, CA, USA

Aenor J. Sawyer

Director, UCSF Skeletal Health Service

Assistant Clinical Professor

Department of Orthopaedic Surgery

University of California

San Francisco

\author{
Laura K. Bachrach \\ Department of Pediatrics \\ Stanford University School of Medicine \\ Stanford, CA, USA
}

ISBN 978-3-319-30410-6 ISBN 978-3-319-30412-0 (eBook)

DOI 10.1007/978-3-319-30412-0

Library of Congress Control Number: 2016946355

(c) Springer International Publishing Switzerland 2007, 2016

This work is subject to copyright. All rights are reserved by the Publisher, whether the whole or part of the material is concerned, specifically the rights of translation, reprinting, reuse of illustrations, recitation, broadcasting, reproduction on microfilms or in any other physical way, and transmission or information storage and retrieval, electronic adaptation, computer software, or by similar or dissimilar methodology now known or hereafter developed.

The use of general descriptive names, registered names, trademarks, service marks, etc. in this publication does not imply, even in the absence of a specific statement, that such names are exempt from the relevant protective laws and regulations and therefore free for general use.

The publisher, the authors and the editors are safe to assume that the advice and information in this book are believed to be true and accurate at the date of publication. Neither the publisher nor the authors or the editors give a warranty, express or implied, with respect to the material contained herein or for any errors or omissions that may have been made.

Printed on acid-free paper

This Springer imprint is published by Springer Nature

The registered company is Springer International Publishing AG Switzerland 


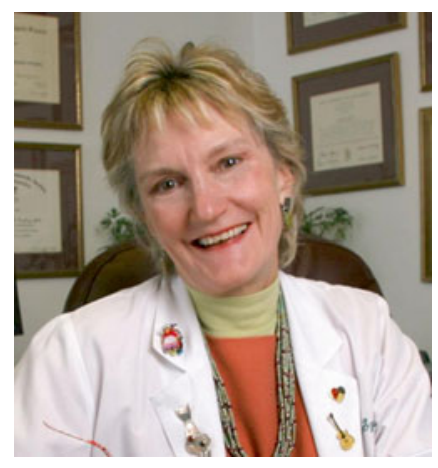

This second edition of Bone Health Assessment in Pediatrics: Guidelines for Clinical Practice is dedicated to Dr. Elizabeth Szalay, one of our coauthors and most respected colleagues who passed away in December 2014. She was a skilled surgeon, clinician, researcher, and educator in the field of pediatric orthopedic surgery. She not only transformed the lives of many children but her warmth, enthusiasm, and generous mentorship inspired numerous trainees under her leadership. Dr. Szalay was a devoted advocate for underserved youth-Native American children, children with physical disabilities, and those living in extreme poverty. She worked tirelessly to optimize the quality of life for her patients and their families. It is with tremendous respect that her writing is memorialized in this text. We, among many, are very grateful for her unparalleled contributions to healthcare.

Ellen B. Fung, Ph.D., R.D., C.C.D. Laura K. Bachrach, M.D. Aenor J. Sawyer, M.D. 



\section{Foreword}

Over the past 20 years, the high interest in pediatric bone disease and the growing body of knowledge on skeletal development have led to the delineation of a new field where clinicians and investigators devote their efforts to the understanding, evaluation and treatment of bone diseases in children. Great momentum was obtained by the development of new non-invasive technologies, and among them densitometry. Such measurements in adult subjects have for a long time brought about unique information with high precision and reproducibility. Their major limitation however is that density results are expressed in two dimensions and extrapolated to represent volumetric density in a 3D bone structure. This is adequate if the size and shape of the studied bone are stable over time. It clearly does not apply to the bones of a growing child wheresize and shape change continuously until growth plates are fused. Thus two-dimensional DXA results had to be adapted to reflect this reality. This challenge has been met as described in details in the first chapters of this book. Interpretation of DXA data using correction parameters to take into account age, sex and body size have generated graphs and tables that are now integral parts of the evaluation of various conditions where bone development and structure are affected. As progress was made, new techniques have emerged that allow for three-dimensional imaging such as QCT, pQCT and HR-pQCT. Now trabecular and cortical bone compartments can be analyzed separately and bone formation evaluated with precision. It may become the equivalent of a non-invasive bone biopsy. Changes in cortical porosity, not captured by DXA will become an important end point in several studies. The only limitation of HR-pQCT is that it can only evaluate appendicular sites due to the amount of irradiation it generates and the design of the equipment. But this also may change in the future. All these considerations and more are covered in details in this Second Edition of Bone Health Assessment in Pediatrics: Guidelines for Clinical Practice. As such it represents an indispensable source of information and guidance for any clinician dealing with pediatric bone diseases.

Montreal, QC, Canada

Francis H. Glorieux, OC, MD, PhD 



\section{Preface}

Eight years ago, we published the textbook Bone Densitometry in Growing Patients to assist clinicians in evaluating bone health in children and adolescents. Since 2007, the field of pediatric bone densitometry has changed dramatically. Despite the emergence of alternative imaging devices, dual energy x-ray absorptiometry (DXA) remains the gold standard method for skeletal assessments in clinical practice. As such, we felt it was important to address some of the changes and new directions in the field with the second edition of the text, slightly modified in title to: Bone Health Assessment in Pediatrics: Guidelines for Clinical Practice.

Skeletal health determined in childhood and adolescence influences an individual's lifetime risk of bone fragility. Peak bone mass reached by early adulthood represents the "bone bank" for life. For this reason, optimizing bone acquisition in the first two decades can help prevent osteoporosis. As this awareness of the importance of early bone health has grown over the past decade, so has the concern for young patients facing threats to bone acquisition. These observations have led to greater demands for diagnostic and therapeutic tools to address bone fragility in children and adolescents.

Many of the chapter authors in this text have spent the past decade improving the ability to accurately assess pediatric bone health by DXA through the collection of, large and robust, ethnic-specific reference data sets. Moreover in October, 2013, many of these same individuals came together for the 2nd Pediatric Position Development Conference to draft what are now the 2013 International Society for Clinical Densitometry (ISCD) Guidelines for Pediatric DXA assessment, interpretation, and reporting. As part of these guidelines, a new definition of osteoporosis in pediatrics was adopted, and the relationship between DXA and fracture prediction clarified.

With all marked changes in the field since the last edition of this text, it was difficult to limit the discussion to 13 short chapters. Those that are included were considered to be the most relevant to the practicing pediatrician. Some of the highlights of this edition include an entire chapter on the assessment of infants and toddlers, a chapter devoted to the assessment of children with disabling conditions, 
an in-depth discussion of vertebral fracture and its etiologies, and a thorough review of the advantages and limitations of densitometry techniques including DXA, pQCT, HRpQCT, and MRI. New fracture prediction software including Trabecular Bone Score and Finite Element Analysis are described. In this edition, the limitations of DXA are addressed as are the most recent strategies for handling them including proposed DXA adjustments such as height Z-score. Our overarching goal is to provide the basic analysis and evaluation tools necessary for clinicians to optimize bone health for all children especially those with skeletal fragility.

This second edition is designed to provide distilled but multidimensional perspectives needed by clinicians interested in bone health. It is anticipated that those who work with the most challenging patients need practical guidance on how to measure and report on their bone health. Given that DXA will likely remain the recommended clinical method to clinically monitor bone health for the foreseeable future, this text can provide useful tools, images, and calculations necessary to be successful.

Oakland, CA, USA

Ellen B. Fung

Palo Alto, CA, USA

San Francisco, CA, USA

Laura K. Bachrach

Aenor J. Sawyer 


\section{Acknowledgments}

This book is the culmination of work from dedicated experts in the field of pediatric densitometry. First and foremost we owe our deepest gratitude to the chapter authors who took time out of their busy schedules to share their knowledge and expertise in this second edition of Bone Health Assessment in Pediatrics: Guidelines for Clinical Practice.

It has been an honor and pleasure to work with many of these authors for a second time. It has also been truly inspiring to engage with the rising stars in this field who are new contributors to this text. The authors' commitment to this work over the past 18 months, despite many professional and personal demands, reflects their deep dedication to improving the skeletal health of all children and adults.

We are grateful for the enduring support and invaluable input from our mentors: Mary Bouxsein, Roland Fischer, Francis Glorieux, Paul Harmatz, James Kasser, Janet King, Mary Leonard, Bertram Lubin, Robert Marcus, Dolores Shoback, Virginia Stallings, Thomas P. Vail, Elliott Vichinsky, Babette Zemel, and our tireless colleagues Richard Capra, Lisa Calvelli, and Marcela Weyhmiller.

In the publishing arena, we thank Michael Griffin, our copy editor, for his patience and endurance with this text, as well as Richard Lansing from Springer Publishing Group for his foresight to consider a second edition of this unique text.

Despite all of the resources, talent, and commitment, we would not have been able to make this second edition a reality without generous support and sponsorship. We would like to thank the S.D. Bechtel Jr. Foundation, the major sponsor of this project and a decade of research and education in the Pediatric Bone Health Consortium. Last, but certainly not least, we are deeply indebted to our families for their patience, support, and unconditional love. We ask for their forgiveness for the many distracted evenings in front of the computer when we could have spent more focused time with them. They provide each of us the creativity, strength, and encouragement on a daily basis, without which we would not have had the inspiration or energy needed to accomplish this work.

Ellen B. Fung, Ph.D., R.D., C.C.D.

Laura K. Bachrach, M.D. Aenor J. Sawyer, M.D. 



\section{Contents}

1 Rationale for Bone Health Assessment in Childhood and Adolescence

Maria Luisa Bianchi, Aenor J. Sawyer, and Laura K. Bachrach

2 Tools for Measuring Bone in Children and Adolescents

Kate A. Ward, Thomas M. Link, and Judith E. Adams

3 Dual-Energy X-Ray Absorptiomery Technology

John Shepherd and Nicola J. Crabtree

4 Indications for DXA in Children and Adolescents

Sarah Pitts and Catherine M. Gordon

5 Acquisition of DXA in Children and Adolescents

Nicola J. Crabtree and Kyla Kent

6 Analysis and Evaluation of DXA in Children and Adolescents.

Babette S. Zemel and Heidi J. Kalkwarf

7 Reporting DXA Results for Children and Adolescents

Ellen B. Fung

8 DXA Evaluation of Infants and Toddlers

Bonny L. Specker and Teresa L. Binkley

9 DXA in Children with Special Needs

Heidi H. Kecskemethy, Elizabeth Szalay, and H. Theodore Harcke

10 Bone Mineral Density as a Predictor of Vertebral Fractures in Children and Adolescents

Leanne M. Ward and Jinhui Ma

11 Lessons Learned from Clinical Research Using QCT, pQCT, and HR-pQCT

Heather M. Macdonald, Heather A. McKay, and Mary B. Leonard 
12 Evaluation of Fracture Without Known Trauma:

Use of DXA in Differential Diagnosis.

M. Zulf Mughal, Amanda T. Whitaker, and Aenor J. Sawyer

13 What's Next in the Field of Bone Health in Pediatrics?

Research Considerations

Sharmila Majumdar, Tony M. Keavney, Luis Del Rio,

Oliver Semler, and Renaud Winzenrieth

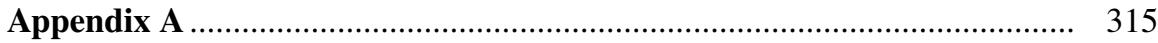

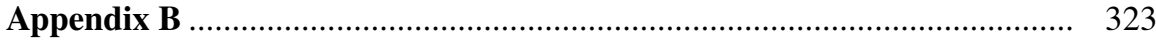

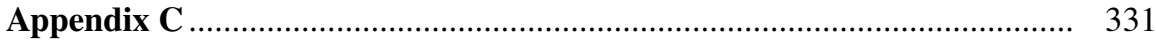

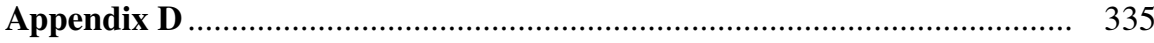

Index 


\section{Contributors}

Judith E. Adams, M.B.B.S., F.R.C.R., F.R.C.P. Radiology \& Manchester Academic Health Science Centre, Central Manchester University Hospitals NHS Foundation Trust, University of Manchester, Manchester, Lancashire, UK

Laura K. Bachrach, M.D. Department of Pediatrics, Stanford University School of Medicine, Stanford, CA, USA

Maria Luisa Bianchi, M.D. Experimental Laboratory for Children's Bone Metabolism Research, Bone Metabolism Unit, Istituto Auxologico Italiano IRCCS, Milan, Italy

Teresa L. Binkley, Ph.D. EA Martin Program, South Dakota State University, North Brookings, SD, USA

Nicola J. Crabtree, Ph.D. Department of Endocrinology, Birmingham Children's Hospital, Birmingham, West Midlands, UK

Ellen B. Fung, Ph.D., R.D., C.C.D. UCSF Benioff Children's Hospital, Oakland, CA, USA

Catherine M. Gordon, M.D., M.Sc. Division of Adolescent and Transition Medicine, Cincinnati Children's Hospital Medical Center, University of Cincinnati College of Medicine, Cincinnati, OH, USA

H. Theodore Harcke, M.D., F.A.C.R., F.A.I.U.M. Department of Medical Imaging, Nemours/A.I. duPont Hospital for Children, Wilmington, DE, USA

Heidi J. Kalkwarf, Ph.D. Gastroenterology, Hepatology and Nutrition, Cincinnati Children's Hospital, Cincinnati, OH, USA

Tony M. Keavney, Ph.D. Mechanical Engineering and Bioengineering, University of California, Berkeley, CA, USA 
Heidi H. Kecskemethy, M.S.Ed., R.D.N., C.S.P., C.B.D.T. Departments of Biomedical Research and Medical Imaging, Nemours/A.I. duPont Hospital for Children, Wilmington, DE, USA

Kyla Kent, B.A., C.B.D.T. Stanford School of Medicine, Palo Alto, CA, USA

Mary B. Leonard, M.D., M.S.C.E. Department of Pediatrics, Stanford University School of Medicine, Stanford, CA, USA

Thomas M. Link, M.D., Ph.D. Department of Radiology and Biomedical Imaging, UCSF, San Francisco, CA, USA

Jinhui Ma, Ph.D. School of Epidemiology, Public Health and Preventive Medicine, University of Ottawa, Children's Hospital of Eastern Ontario Research Institute, Ottawa, ON, Canada

Heather M. Macdonald, Ph.D. Department of Family Practice and Centre for Hip Health and Mobility, University of British Columbia, Vancouver, BC, Canada

Sharmila Majumdar, Ph.D. University of California, San Francisco, CA, USA

Heather A. McKay, Ph.D. Departments of Orthopaedics and Family Practice, Centre for Hip Health and Mobility, Vancouver, BC, Canada

M. Zulf Mughal, M.B., Ch.B., F.R.C.P. Department of Paediatric Endocrinology, Royal Manchester Children's Hospital, Manchester, UK

Sarah Pitts, M.D. Divisions of Adolescent Medicine and Endocrinology, Harvard Medical School, Boston Children's Hospital, Boston, MA, USA

Luis Del Rio, M.D., C.C.D. Department of Bone Densitometry, Hospital Sant Joan De Deu, Barcelona, Spain

Aenor J. Sawyer, M.D., M.S. Director, UCSF Skeletal Health Service, Assistant Clinical Professor, Department of Orthopaedic Surgery, University of California, San Francisco

Oliver Semler, M.D. Department of Rare Skeletal Disease, Children's Hospital, University of Cologne, Cologne, Germany

John Shepherd, Ph.D. Department of Radiology and Biomedical Imaging, University of California, San Francisco, CA, USA

Bonny L. Specker, Ph.D. EA Martin Program, South Dakota State University, North Brookings, SD, USA

Elizabeth Szalay, M.D. (Deceased) Pediatric Orthopedic Surgery, Carrie Tingley Hospital, University of New Mexico, Albuquerque, NM, USA

Kate A. Ward, Ph.D. MRC Human Nutrition Research, Cambridge, Cambridge CB1 9NL and MRC Lifecourse Epidemiology Unit, University of Southampton, Southampton, UK 
Leanne M. Ward, M.D., F.A.A.P., F.R.C.P.C. Division of Endocrinology and Metabolism, Department of Pediatrics, Children's Hospital of Eastern Ontario, University of Ottawa, Ottawa, ON, Canada

Amanda T. Whitaker, M.D. Department of Orthopaedic Surgery, Boston Children's Hospital, Boston, MA, USA

Renaud Winzenrieth, Ph.D. Research and Development Department, Medimaps SASU, Merignac, France

Babette S. Zemel, Ph.D. Department of Pediatrics, The Children's Hospital of Philadelphia, Philadelphia, PA, USA 